Presented at the IEEE Nuclear Science Symposium, Norfolk, Virgina, Oct. 30-Nov.5, 1994.

(To be published in IEEE Trans. Nucl. Sci.)

WIS-94/47/OCT.-PH

\title{
New Ideas in CsI-Based Photon Detectors: Wire Photomultipliers and Protection of the Photocathodes
}

\author{
A.Breskin, A.Buzulutskov * and R.Chechik \\ Department of Particle Physics, The Weizmann Institute of Science, 76100 Rehovot, Israel
}

\begin{abstract}
We discuss the idea of a vacuum or gaseous photomultiplier with a CsI-coated wire photocathode. It benefits of an electric field enhanced quantum efficiency. We present a method for protection air-sensitive photocathodes with thin dielectric film coating. Results of CsI coating with thin $\mathrm{LiF}$ and $\mathrm{MgF}_{2}$ films are shown. The protection of other UV and visible photocathodes is discussed.
\end{abstract}

\section{INTRODUCTION}

During last years one can observe a growing interest in photon detectors based on both photoelectric $[1,2]$ and secondary electron emission (SEE) [3] from CsI films. CsI solid convertors have a relatively good short-term stability in air [1], high quantum efficiency (QE) in the far UV region and good radiation hardness [4]. CsI also provides high radiation-induced secondary electron vield [5].

These properties allow one to consider the CsI-based gaseous photomultiplier as an alternative imaging detector for Ring Imaging Cherenkov (RICH) devices, substituting TMAE vapour-based photon detectors [6]. There is already some evidence to the medium-term stability of CsI photocathodes in presence of chamber gases and under gas multiplication conditions [7], however the long-term stability is of prime importance when operating solid photocathodes in large gaseous wire chambers.

Another promising application of CsI-based wire chambers could be in the field of crystal calorimetry and PET. Such devices have a subnanosecond time resolution [8] and a red boundary cutoff suitable for the detection of the fast emission component of the $\mathrm{BaF}_{2}$ scintillator. The CsI photocathode is insensitive to the slow component of $\mathrm{BaF}_{2}$, which is of prime importance in these applications [9]. These latter applications however are hindered by the relatively low $\mathrm{QE}$ of $\mathrm{CsI}$ in the $\mathrm{BaF}_{2}$ fast emission region, i.e. above $190 \mathrm{~nm}$.

In the present work we discuss new, rather exotic, ideas regarding the protection of the photocathodes and the en-

\footnotetext{
*n leave of absence from IHEP, Protvino, Russia
}

hancement of their response. The problem of the low QE of CsI at longer wavelengths could be overcome by applying a high electric field at the photocathode surface, using for example a wire photocathode structure. This wire photomultiplier approach is based on our recent results on the photoelectric and secondary electron emission from CsI at very high electric fields $[10,11]$.

Long-term stability of the photoconvertor could be reached by coating its surface with a thin dielectric protection film. Interesting effects have been revealed during this study, in particular a reduction of the photocathode electron affinity due to a polarized adsorption of water molecules. We consider the results on the CsI protection studies as a testing ground for our further application to the protection of air-insensitive visible photocathodes.

\section{WIRE PHOTOMULTIPLIERS}

A. Field enhancement of the photoelectric emission from CsI

To obtain high electric fileds on the photocathode surface we used a multiwire plane cathode, placed between twc anode planes (Fig.1) [10]. The cathode grid was made from wires of $10 \mu \mathrm{m}$ in diameter with a $1 \mathrm{~mm}$ spacing. The wire photocathode was prepared by vacuum evaporation of a CsI layer on one side of the grid. The CsI layer had a crescent moon shape with a maximum thickness of $500 \mathrm{~nm}$. Fig.2a shows the dependence of the $\mathrm{QE}$ of CsI, in vacuum, on the field at wavelengths of $160 \mathrm{~nm}, 185 \mathrm{~nm}$ and $\geq 200$ $\mathrm{nm}[10]$. The data are normalized to the $\mathrm{QE}$ measured at $1 \mathrm{kV} / \mathrm{cm}$. One can see a dramatic dependence of the QE on the field: at a field of $500 \mathrm{kV} / \mathrm{cm}$ the enhancement factor of the photoyield is 1.5 at $160 \mathrm{~nm}, 3$ at $185 \mathrm{~nm}$ and 25 at $\geq 200 \mathrm{~nm}$. We explain the field enhancement by a decrease of the CsI electron affinity due to the Schottky effect applied to insulators:

$$
\Delta E_{a}=e(\alpha e F)^{1 / 2} ; \quad \alpha=\left(\epsilon_{\infty}-1\right) /\left(\epsilon_{\infty}+1\right)
$$

Here $\Delta E_{a}$ is the field-induced electron affinity decrease $\epsilon_{\infty}$ the high-frequency dielectric constant, $e$ is the electron charge, $F$ the electric field strength. 


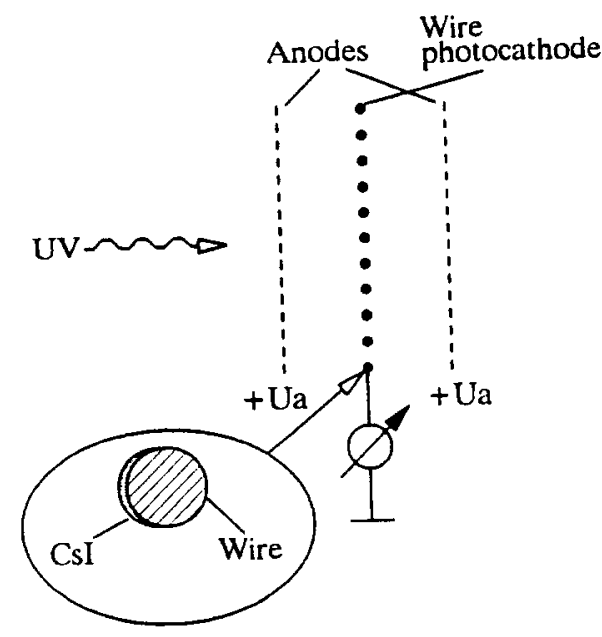

Figure 1: A schematic view of the experimental set-up for the study of photoemission from CsI at high electric fields.

One should notice the unique opportunity to observe a field enhancement effect in CsI at relatively low fields, up to $500 \mathrm{kV} / \mathrm{cm}$, due to a very low electron affinity of CsI: $E_{a}=0.2 \mathrm{eV}$. For most insulators the affinity is of the order of $1 \mathrm{eV}$, resulting in $(1 / 0.2)^{2}=25$ times higher fields required to make the effect observable.

\section{B. Field enhanced response to $B a F_{2}$ scintillations}

As one can see from Fig.2a, the field enhancement is large for longer wavelengths, which makes it very attractive for the enhancement of the CsI response to $\mathrm{BaF}_{2}$ scintillations. Indeed, the emission spectrum of the $\mathrm{BaF}_{2}$ fast component coincides with the long-wavelength tail of the $\mathrm{QE}$ curve of CsI. The enhancement of the CsI response to $\mathrm{BaF}_{2}$ scintillations was demonstrated in ref. [11]: the CsI wire photocathode, shown in Fig.1, was coupled to $\mathrm{BaF}_{2}$ or $\mathrm{KMgF}_{3}$ crystals, irradiated with a strong $\gamma$-ray source. Fig. $2 \mathrm{~b}$ shows a normalized photocurrent from the CsI wire photocathode as a function of the field strength. The field enhancement effect for $\mathrm{BaF}_{2}$ is much stronger compared to that of $\mathrm{KMgF}_{3}$ due to its longer wavelength emission, and reaches a factor of 3 at $400 \mathrm{kV} / \mathrm{cm}$.

The data presented in Fig.2a,b were obtained with a CsI photocathode that was not subjected to a vacuum thermal treatment following its production. It is known however that the CsI photoresponse can be considerably enhanced by heating the photocathode following its preparation [12, 13]. Fig. 3 gives an example of the heat enhancement effect, observed for a CsI photocathode evaporated on a stainless steel plate substrate. The absolute QE spectra are shown at different times after the evaporation, the temperature of the substrate being sustained at $50^{\circ} \mathrm{C}$. Simalar to the field enhancement, the heat treatment considerably extends the long-wavelength range of the $\mathrm{QE}$ spectrum.

The combination of the heat and field enhancement is therefore supposed to provide a superior sensitivity to the
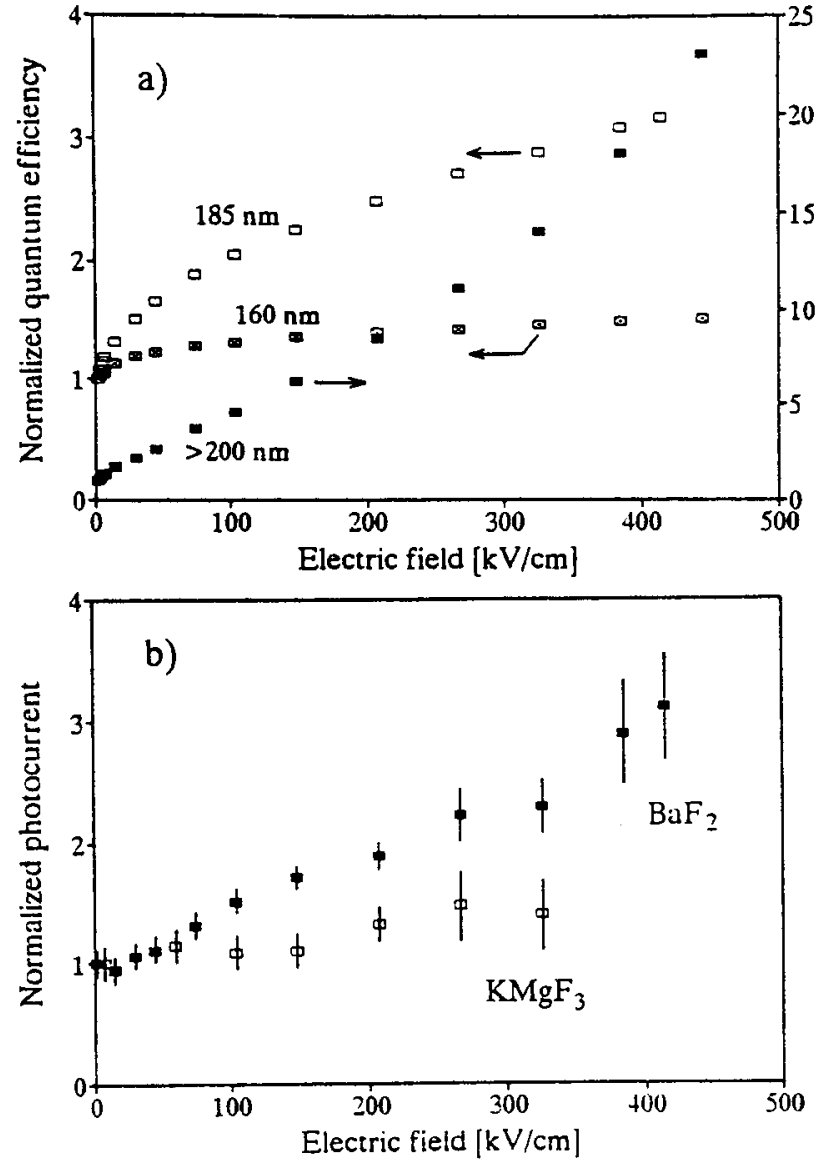

Figure 2: a) Normalized quantum efficiency of CsI as a function of the electric field strength, at 160,185 and $\geq 200 \mathrm{~nm}$. Each data set is normalized to the point at a field value of about $1 \mathrm{kV} / \mathrm{cm}$. b) Normalized photocurrent from CsI as a function of the electric field strength for $\mathrm{BaF}_{2}$ and $\mathrm{KMgF}_{3}$ scintillations.

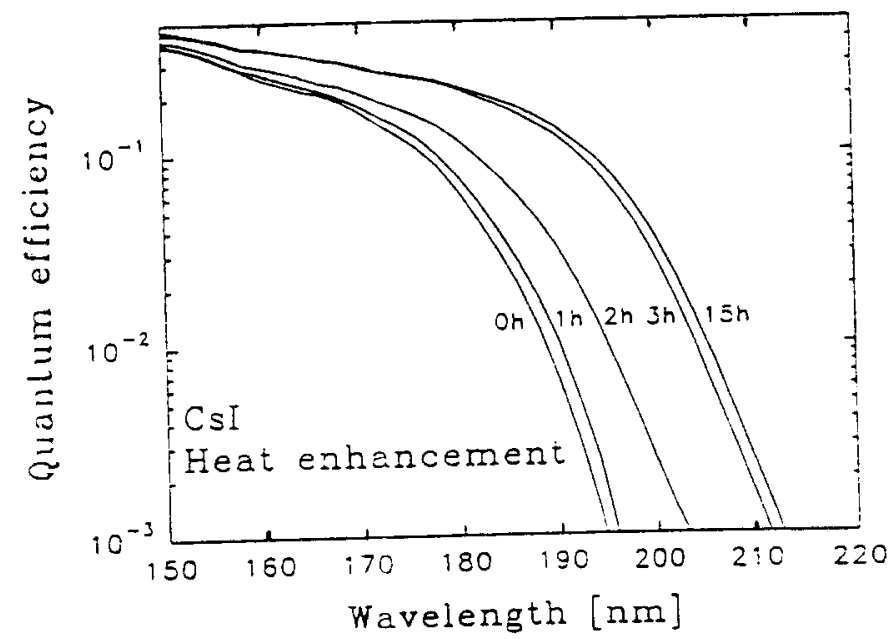

Figure 3: Quantum efficiency spectra of CsI at different heating times after the evaporation. 


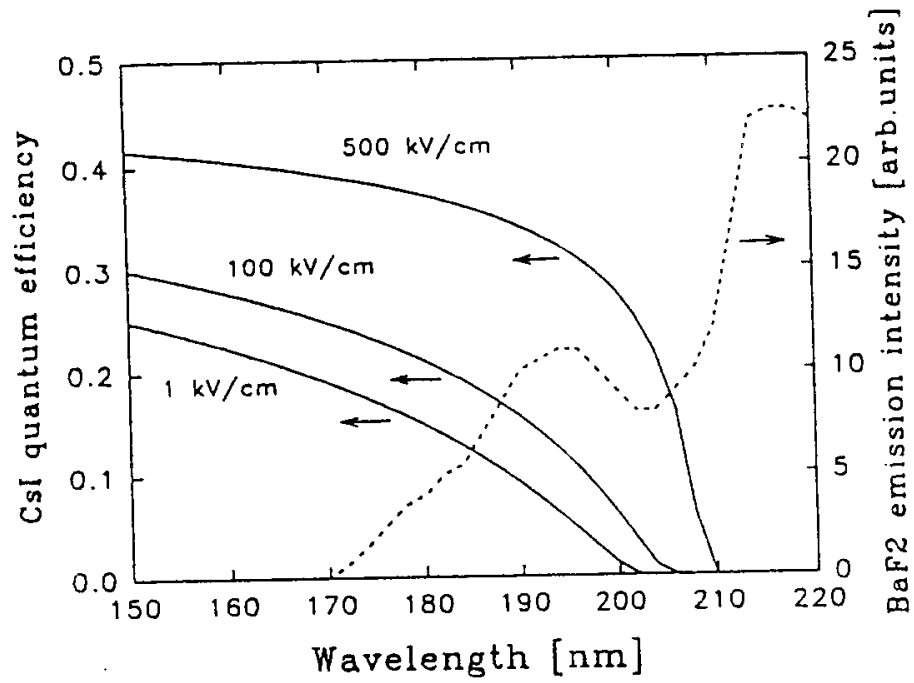

Figure 4: Theoretical QE spectra of CsI at different electric fields. The emission spectrum of the fast scintillation component of $\mathrm{BaF}_{2}$ is shown as well.

$\mathrm{BaF}_{2}$ fast scintillation component. We carried out some calculations of the field enhancement of the $\mathrm{QE}$ of a heattreated photocathode, using a simple model described elsewhere [10]. Fig.4 shows the expected QE spectrum of CsI at field strengths of 1,100 and $500 \mathrm{kV} / \mathrm{cm}$, calculated using expression (9) from that ref. One can observe that the response of the heat-treated CsI photocathode to the $\mathrm{BaF}_{2}$ scintillation can be enhanced by a factor of 4 at a field of $500 \mathrm{kV} / \mathrm{cm}$. Taking into account the absolute light yield of the fast emission component of $\mathrm{BaF}_{2}(2000$ photons/MeV [9]), we estimate a maximum attainable response of a heat- and field-enhanced CsI photocathode to $\mathrm{BaF}_{2}$ scintillation to be of the order of 40 photoelectrons per $1 \mathrm{MeV}$ of deposited energy. This should be compared to about 20 photoelectrons/MeV measured with a TMAE vapour photocathode [14].

\section{Field enhancement of the secondary electron emission from CsI}

We have studied the dependence of the electron-induced secondary electron emission (SEE) yield of CsI as a function of the electric field and primary electron energy, using a CsI wire cathode similar to that used in the UV experiment [10] (see Fig.4,5 in that ref.). The general results are the following. For low primary electron energies, of the order of $100 \mathrm{eV}$, the SEE yield is independent of the field. In the primary electron energy range of 1-4 keV the SEE yield rapidly increases by a factor of $2-5$, when increasing the field from 1 to $15 \mathrm{kV} / \mathrm{cm}$. With a further field increase, the SEE yield for these electron energies, grows much slower and even tends to saturate for the lower electron energies.

In this experiment a relative $\mathrm{SEE}$ yield was measured. If to normalize our relative SEE data measured at low field, to the absolute SEE ones measured also at low field el- swhere [15], one can deduce absolute values of the SEE yield at high electric fields. At a primary electron energy of $2 \mathrm{keV}$ and at a field above $150 \mathrm{kV} / \mathrm{cm}$ we estimate that about 40 secondary electrons are emitted per incident electron.

\section{A wire photomultiplier}

The efficient area of the wire photocathode discussed above is very low, about $1 \%$ of the total surface, due to the low diameter-to-wire spacing ratio. This was needed in order to obtain a high electric field at the wire surface, in this particular wire-cell geometry. In fact the geometry of the cell used is the one of a classical multiwire proportional chamber, but with reverse electrode polarity (see Fig.1). Covering the detection area with a stack of such wire-cells seems to be unpractical due to a large number of cells needed.

In order to have a practical device, which would assure a reasonable area efficiency, the solution of a complex 3-dimensional geometrical and electrostatical problem of wire packing is required. The way of multiplying photoelectrons released from the photocathode wires is important as well. In some aspects the problem of wire packing resembles that of sphere packing, not yet mathematically solved in $\geq 3$ dimensions [16]

We suggest below two designs for a wire photocathode cell geometry. To efficiently cover a given detection area, the wire-cells are assumed to be placed in series. The first design is for the case of a wire photomultiplier operated in a gas multiplication mode (Fig.5a) and the second is for vacuum amplification (Fig.5b). In both cases the wire-cell consists of a single wire plane, with an alternating wire polarity combined in such a way as to make the plane electricaly neutral, namely independent of the neighboring planes. This condition can be reached if the planes are placed at a distance larger than the wire spacing, where the electric field of a single plane approaches zero. In the first case this is achieved by a wire polarization sequence $+-+-+-\ldots$, in the second case by $+0-0+0-0+0-0 \ldots$ sequence.

For the first, simplest wire-cell geometry (Fig.5a) we calculated the electric field $F$ at the cathode wire surface in a complex potential approach [17]:

$$
F=2 C U / r ; \quad C=1 /\left(2 \ln \frac{s-r}{r}\right)
$$

Here $r$ and $s$ are the wire radius and spacing respectively, $C$ the wire capacitance per unit length, $U$ the voltage difference between the anode and cathode wires. For a $50 \mu \mathrm{m}$ wire diameter and $0.5 \mathrm{~mm}$ wire spacing, providing $5 \%$ photocathode area efficincy per one wire-cell, this expression gives a field value $F=407 \mathrm{kV} / \mathrm{cm}$ at $U=3 \mathrm{kV}$, which is high enough to exploit the field enhancement effect.

A gas multiplication can take place in the vicinity of the anode wires. In order to diminish a contribution of dead zones, the anode wire diameter should be not larger 
than that of the cathode wire. A maximum gas gain and a maximum attainable field on the cathode wire is strongly dependent on many parameters such as the wire diameter, gas mixture, gas pressure etc. For example, the results with a thin multiwire chamber with a similar geometry obtained elsewhere [18], showed that an electric field of about $350 \mathrm{kV} / \mathrm{cm}$ can be reached on the anode wire before breakdown, in n-pentane-based mixtures. In order to reduce losses in $\mathrm{QE}$ due to elastic backscattering from gas molecules, it is recommended to use $\mathrm{CH}_{4}$-based gas mixtures. This was discussed in detail in a recent work [19].

Multiplication in the vacuum wire-cell (Fig.5b) takes place via a secondary electron emission from a wire dynode coated with a CsI film, placed between the wire photocathode and the anode. The diameters of the dynode and anode wires shown in the figure, are smaller than that of the cathode wire in order to decrease dead space. As it was mentioned in section II.C, the absolute value of the SEE yield from CsI can reach 40 secondary electrons per 2 $\mathrm{keV}$ primary electron. It means that for a voltage of $2 \mathrm{kV}$, applied between the cathode and dynode wires, the gain of such a vacuum cell can be of the order of a few tens. To optimize the wire-cell geometry one needs do some additional simulation work on the electron trajectories.

The minimal number of wire planes needed to fully cover the detection area, for example for a $50 \mu \mathrm{m}$ wire in diameter and $0.5 \mathrm{~mm}$ wire spacing, is equal to 10 . For a distance of $1.5 \mathrm{~mm}$ between the wire planes the detector could be rather compact in depth: its total thickness could be of the order of $1.5 \mathrm{~cm}$.

Some possible wire plane packing geometries are shown in Fig.6. Firstly, a double-plane element is assembled from two wire-cells, rotated by $90^{\circ}$ with respect to each other. Then we consider two variants: a) every other element is shifted by a step equal to the wire diameter, along the diagonal direction; b) it is rotated by a given angle (shown in the figure, by $30^{\circ}$ ). Variant " $b$ " is simpler to realize due to looser tolerance requirements, though it will need more wire planes.

\section{Protection of the PHOTOCATHODES}

\section{A. Experimental procedure}

We consider here a method for photocathode protection with thin dielectric films. The idea to protect visible photocathodes with a thin solid film in order to make them stable against exposure to air or other gas, is not new. Recently some attempts have been made to protect a $\mathrm{CsSb}$ photocathode with a CsI film $[20,21]$. But CsI itself is air sensitive, reacting with atmospheric water vapours. Water molecules are also harmful to alkali metal-based materials, of which visible photocathodes are usually made. Moreover, protection against water molecules is particularly difficult because of their small size, compared to other a)

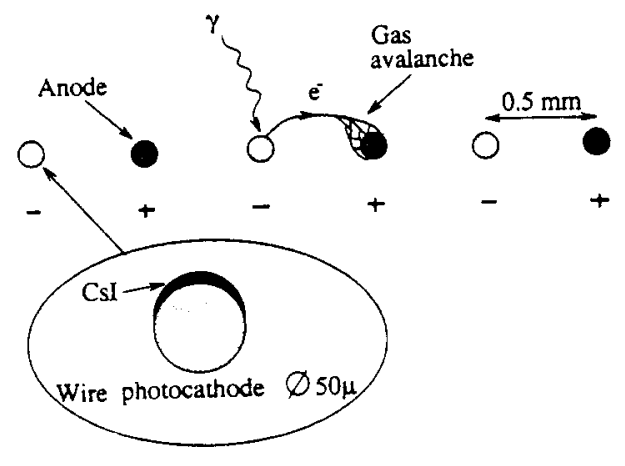

b)

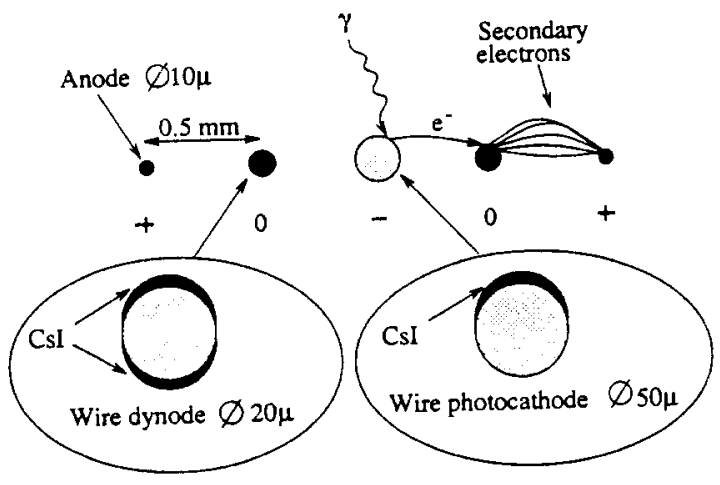

Figure 5: Schematic view of the wire photocathode cell with an amplification in gas (a) and in vacuum (b).

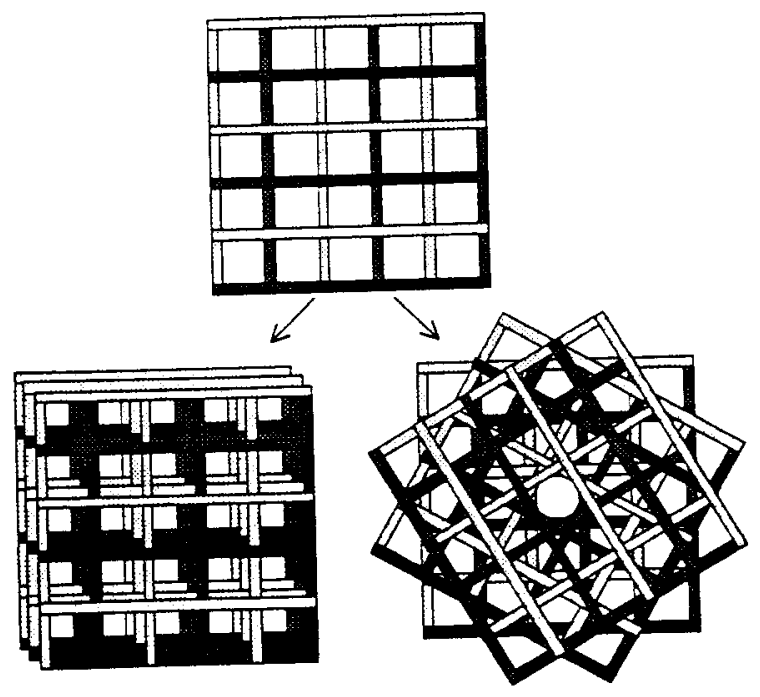

Figure 6: Possible packing procedures to cover the detection area with stacks of wire planes. 
chemically active gases, which is responsible for their high penetration capability. Therefore a protection against water might, in principle, be efficient for other impurities as well.

Below we present the results of the study of CsI photocathodes coated with thin $\mathrm{LiF}$ and $\mathrm{MgF}_{2}$ films and exposed to water vapour. For the preparation of CsI photocathodes a standard procedure was used, including the pre-evaporation of an Al layer on top of the stainless steel substrate, evaporation of $500 \mathrm{~nm}$ of CsI and further heat enhancement treatment. After the stabilization of the $\mathrm{QE}$ at $50^{\circ} \mathrm{C}$, a protection film was evaporated in the same vacuum set-up. Typical vacuum level during these operations was better than $10^{-7}$ Torr.

Two different procedures were used to expose photocathodes to water vapour. In the first one, the vacuum chamber containing the photocathode, was connected to the laboratory air through a small apperture. In the second one, the photocathode was taken out from the chamber and exposed to air in the laboratory. The measured humidity inside and outside the chamber was about $50-55 \%$ and $65-$ $70 \%$ correspondingly. The room temperature was $22^{\circ} \mathrm{C}$. In the following we refer to the first procedure as to "exposure to air", to distinguish from the second one refered to as "exposure to atmosphere".

\section{B. CsI+LiF photocathodes}

Fig.7 shows the QE spectra of a CsI photocathode before and after coating with a $22 \AA$ thick LiF film. After LiF deposition, the $\mathrm{QE}$ decreases by more than one order of magnitude. This decrease is explained by the losses of photoelectrons during their transport through the LiF film.

After exposure to air one can see a dramatic incease of the QE. After further exposure to vacuum this enhancement is conserved, indicating on the stability of the effect. We interpret this effect by a reduction of the $\mathrm{LiF}$ electron affinity, induced by polarized water molecule adsorption on the LiF surface (see Fig.8). Qualitatively, the electron affinity decrease can be explained by a formation of a double-charged layer, resulting in a surface potential jump. Such a double layer structure can be created by an adsorption of polarized molecules with a high dipole moment such as water.

It is interesting to note that the $\mathrm{QE}$ increase after exposure to atmosphere, of a LiF photocathode operated in the extreme VUV region, was reported many years ago [22]. Recently polarized water molecule adsorption on hygroscopic substrates such as $\mathrm{NaCl}$, was proven by a model calculation and was qualitatively supported by experimantal observations using a second optical harmonic generation technique [23]. In our case, due to the slightly hygroscopic character of LiF, the same mechanism may take place. Fig. 8 shows an energy diagram at the CsI/LiF/vacuum interface before and after contact to water vapour. To explain our experimental results, the electron affinity of $\mathrm{LiF}$ should be reduced due to water adsorption, from an initial

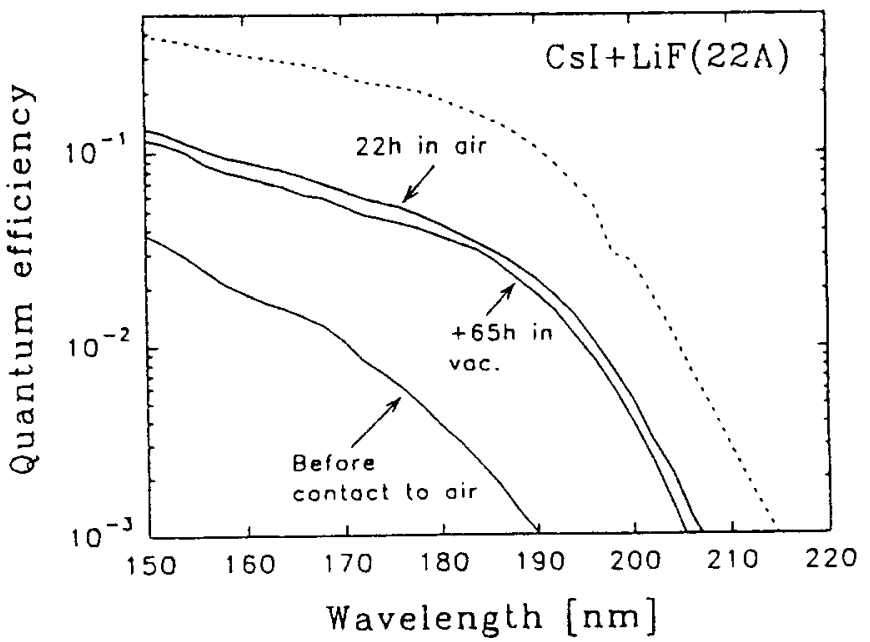

Figure 7: Quantum efficiency spectra of a CsI photocathode coated with a $22 \AA$ thick LiF film, after preparation, after 22 hours exposure to air and after further 65 hours of exposure to vacuum (solid lines). The QE spectrum of the CsI photocathode before coating is shown as well (dashed line).

value of $1 \mathrm{eV}$ to about $0.15 \mathrm{eV}$ [24]. Note that the water layer formed on the photocathode surface is fairly stable: it does not dissappear even after two days of storage in high vacuum.

\section{C. $C s I+M g F_{2}$ photocathodes}

Fig.9 shows the QE spectra of a CsI photocathode coated with of a $22 \AA$ thick $\mathrm{MgF}_{2}$ film. Similar to $\mathrm{LiF}$, after the deposition of the $\mathrm{MgF}_{2}$ film the $\mathrm{QE}$ strongly decreases. However, a further heating of the substrate at $50^{\circ} \mathrm{C}$ significantly improves the $\mathrm{QE}$. We relate this improvement to the thermal annealing of the structural defects in the $\mathrm{MgF}_{2}$ film (the defects are known to trap low energy electrons). No significant water adsorption effect, like in the case of LiF, was observed. This could be due to the nonhygroscopic nature of $\mathrm{MgF}_{2}$.

Fig.10 illustrates the protection capability of the $\mathrm{MgF}_{2}$ film on CsI, after the heat treatment; the QE spectra were measured before and after exposure to atmosphere. For comparison, the QE spectra of the uncoated CsI are shown as well. While the $\mathrm{QE}$ of the uncoated CsI decreases with exposure time to atmosphere, the $\mathrm{QE}$ of CsI coated with $\mathrm{MgF}_{2}$ almost does not change. One may conclude that a $\mathrm{MgF}_{2}$ film of a thickness of a few tens of $\AA$ can protect the photocathode sufficiently well against humidity.

\section{Application to UV and visible photocathodes}

The positive results obtained so far suggest the application of this protection technique to other photocathodes sensitive in the UV and in the visible. Indeed, if to look at Fig.8, one can notice that the LiF film, with an adsorbed 


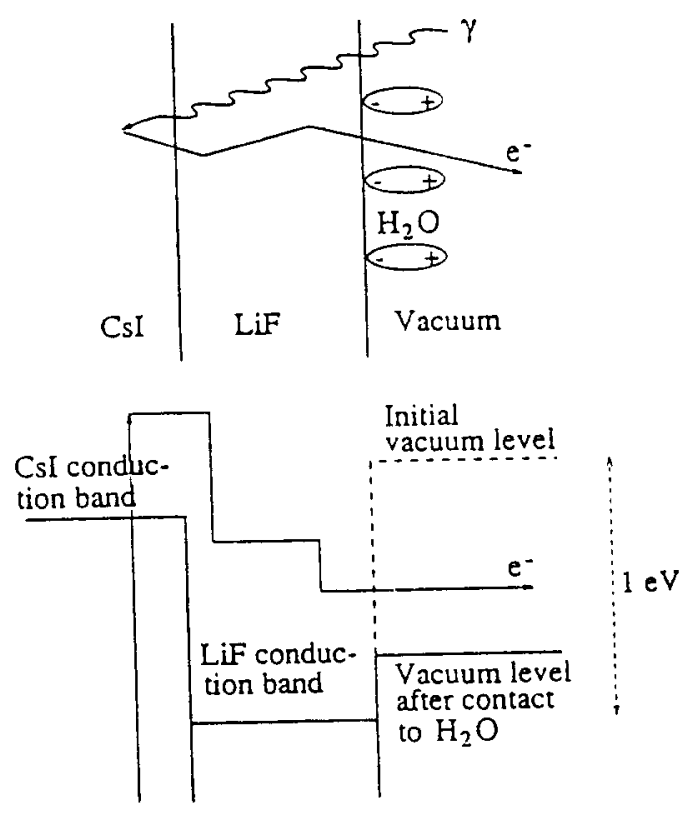

Figure 8: Energy diagram at the CsI/LiF/vacuum interface before and after contact to water vapour (bottom). A schematic picture of the photoemission process is shown as well (top).

water layer on it, reduces the electron affinity of the CsI photocathode by about $1 \mathrm{eV}$. This principle can be applied to other photocathodes as well. The decrease of the electron affinity shifts the red boundary to longer wavelengths. For example, a CuI photocathode coated with LiF and treated with water vapour, seems to be a good candidate. $\mathrm{CuI}$ is stable in gases [25], has a small energy gap $(3 \mathrm{eV})$, but a rather large electron affinity, about 3 $\mathrm{eV}$, resulting in a cutoff near $200 \mathrm{~nm}$ [26]. The reduction of the electron affinity of $\mathrm{CuI}$ with the help of $\mathrm{LiF} /$ water layers would make it sensitive up to about $240 \mathrm{~nm}$.

As far as $\mathrm{MgF}_{2}$ is concerned, one could exploit its high protective property. We tried to estimate the QE spectra of the common visible photocathodes, such as CsSb and $\mathrm{CsNaKSb}$, coated with a $22 \AA$ thick $\mathrm{MgF}_{2}$ film, using the experimental data of Fig. 10 from the present work and that of Figs. 27 and 42 from ref. [26], and the expression:

$$
\begin{array}{r}
Q E_{C s S b+M g F_{2}}(\lambda)=k\left(E_{p e}\right) \cdot Q E_{C s S b}\left(E_{p e}\right) \\
k\left(E_{p e}\right)=\frac{Q E_{C s I+M g F_{2}}\left(E_{p e}\right)}{Q E_{C s I}\left(E_{p e}\right)}
\end{array}
$$

Here $\lambda$ is the wavelength; $E_{p e}$ the initial photoelectron energy relative to vacuum level, coresponding to this wavelength; $k\left(E_{p e}\right)$ the attenuation coefficient of the $\mathrm{QE}$ due to the $\mathrm{MgF}_{2}$ film. A conversion between the photoelectron energy and the wavelength for a particular photocathode is provided by the expression: $E_{p e}=h c / \lambda-E_{g}-E_{a}$, where $E_{g}$ and $E_{a}$ are respectively the energy gap and the electron affinity of the photocathode substance.

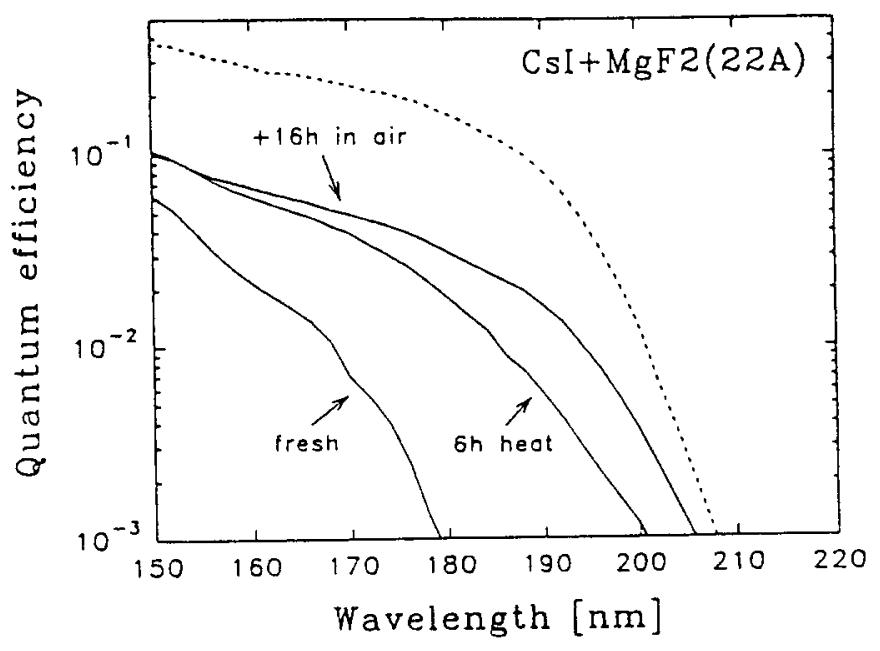

Figure 9: Quantum efficiency spectra of a CsI photocathode coated with a $22 \AA$ thick $\mathrm{MgF}_{2}$ film, after preparation, after 6 hours at $50^{\circ} \mathrm{C}$ and after further 16 hours exposure to air (solid lines). The QE spectrum of the CsI photocathode before coating is shown as well (dashed line).

In the calculations we used an implicit assumption, that the attenuation coefficient in $\mathrm{MgF}_{2}$ for any photocathode is a function of the initial photoelectron energy only, which is generaly not the case due to a possible difference in the energy loss of photoelectrons in CsI and in CsSb. However this difference could not be too large since both photocathodes are good photoemitters, resulting in equally good transport conditions for low energy electrons.

Fig. 11 shows the results of calculations for $\mathrm{CsSb}+\mathrm{MgF}_{2}$ and $\mathrm{CsNaKSb}+\mathrm{MgF}_{2}$ photocathodes. From this figure one could expect the $\mathrm{QE}$ value of the protected photocathodes to be high enough (up to several percents for blue light) for a broad spectrum of potential applications.

\section{CONCLUSIONS}

The new ideas presented here in the field of photon imaging, tend to exploit our recent observations of a field enhanced photoelectric and secondary electron emission, and photocathode protection with thin films.

The electric field effects, which we believe are dependent on the square root of the field strength [10], can be applied in a practical way to photocathodes with rather low electron affinity. These could be for example $\mathrm{CsSb}\left(E_{a}=0.4 j\right.$ $\mathrm{eV}), \mathrm{CsNaKSb}\left(\mathrm{E}_{a}=0.55 \mathrm{eV}\right)$ or photocathodes with negative electron affinity. High electric fields can be attained in wire structures, which suggests the development of wire photomultipliers equiped with wire photocathodes. Obvious advantages of the wire photomultipliers considered. operated in gas or vacuum, are their robustness and lower sensitivity to magnetic fields as compared to normal photomultiplier tubes. Their drawback could be a low gain. though for some applications such as calorimetry, it could 


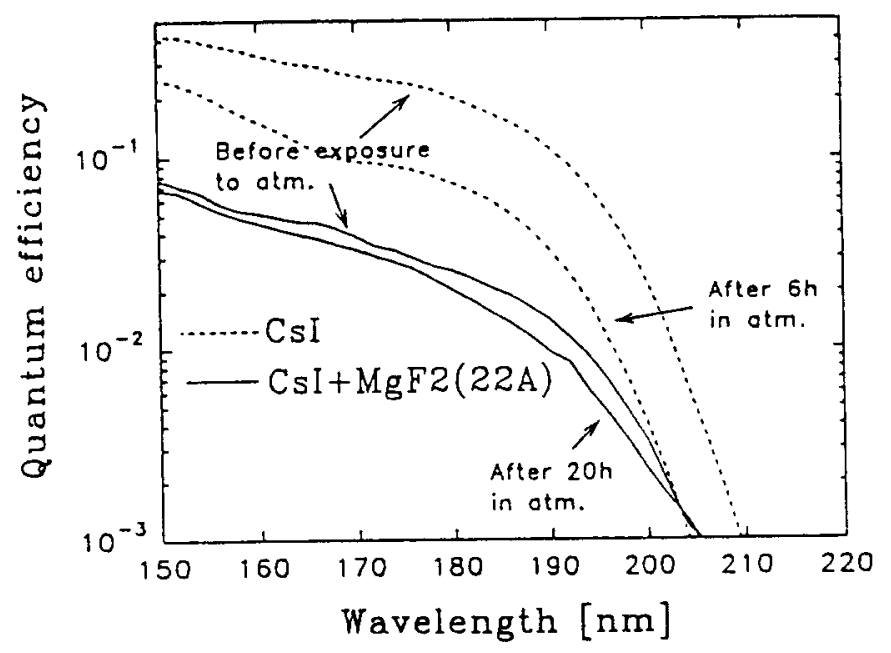

Figure 10: Results of the protection capability of $\mathrm{MgF}_{2}$. Shown are the quantum efficiency spectra of a CsI photocathode coated with a $22 \AA$ thick $\mathrm{MgF}_{2}$ film, heat-treated, before and after 20 hours exposure to atmosphere and of an uncoated CsI photocathode before and after 6 hours exposure to atmosphere.

be sufficient. However, a considerable research work is needed to put this idea into practice. Other methodes of creating high electric fields on flat surfaces (not wires) are investigated by us as well.

A further development of the photocathode protection method with thin films requires a full understanding of the related physics of low energy electron transport in dielectric films and surface phenomena. We consider the prelimanary results obtained here with a CsI photocathode, as very promising. We further exploit this field of protection of photocathodes, with the main goal being to make visible photocathodes operative under gas amplification conditions. This finally could lead to the development of large area alternative photon imaging detectors, capable to compete with vacuum photomultipliers and solid state devices.

\section{ACKNOWLEDGEMENTS}

We would like to thank Dr.A.Akkerman, Mr.T.Boutboul and Dr.J.Va'vra for helpful discussions and Dr.D.Vartsky for his contribution to some of the research work presented here. This work was supported by the Foundation Mordoh Mijan de Salonique, by the Basic Research Foundation of the Israel Academy of Sciences and Humanities, by the United States-Israel Binational Science Foundation (BSF) and by an EEC research grant. A.Buzulutskov is grateful to the Feinberg Graduate School for his support.

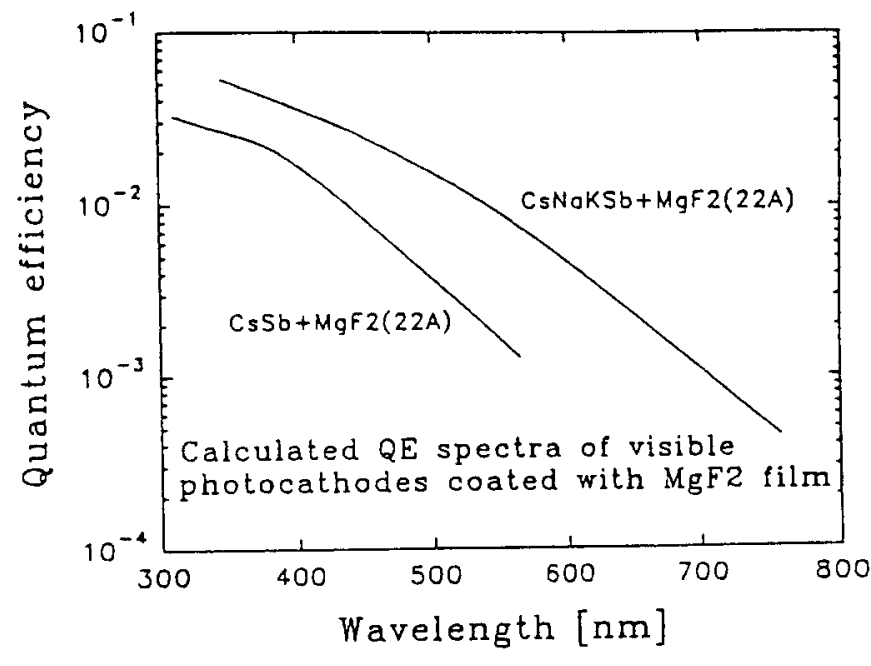

Figure 11: Predicted quantum efficiency spectra of $\mathrm{CsSb}$ and CsNaKSb photocathodes, coated with $22 \AA$ thick $\mathrm{MgF}_{2}$ films.

\section{REFERENCES}

[1] V.Dangendorf, A.Breskin, R.Chechik and H.SchmidtBöcking, Nucl. Instr. and Meth. A289(1990)322.

[2] G.Charpak, V.Peskov, D.Scigocki and V.Valbis, Proc. Int. Simp. on Particle Identification at High Luminosity Hadron Colliders, ed. T.G.Gouraly and I.G.Morfin, (FNAL, IL, USA, 1989), p.295.

[3] A.Breskin,R.Chechik,A.Akkerman,A.Gibrekhterman, V.Elkind, I.Frumkin and A.Notea, Nucl. Instr. and Meth. A348(1994)207.

[4] P.Miné, Nucl. Instr. and Meth. A343(1994)99, and references therein.

[5] S.A.Schwarz, J. Appl. Phys. 68(1990)2382, and references therein.

[6] A.Braem, A.DiMauro, E.Nappi, A.Ijubicic Jr., G.Paic, F.Piuz, F.Posa, R.S.Ribeiro, T.Scognetti and T.D.Williams, Nucl. Instr. and Meth. A343(1994)163.

[7] H.Berger et al., Recent results on the properties of CsI photocathodes, presented at the Int. Conf. on Frontier Detectors for Frontier Physics, May 1994, Elba, Italy, Nucl. Instr. and Meth., in press.

[8] V.Dangendorf, A.Breskin, R.Chechik and H.SchmidtBöcking, Nucl. Instr. and Meth. A308(1991)519.

[9] S.Majewski and C.Zorn, Fast scintillators for high radiation levels, in: Instrumentation in High Energy Physics, ed. F.Sauli, (World Scientific. 1992). p.157, and references therein. 
[10] A.Buzulutskov, A.Breskin and R.Chechik, Field enhancement of the photoelectric and secondary electron emission from CsI, Preprint WIS-94/11/Feb.-PH. Submitted to J. Appl. Phys.

[11] A.Buzulutskov, A.Breskin, R.Chechik and D.Vartsky, Nucl. Instr. and Meth. A350(1994)406.

[12] D.F.Anderson, S.Kwan, V.Peskov and B.Hoeneisen, Nucl. Instr. and Meth. A323(1992)626.

[13] H.Brauning, A.Breskin, R.Chechik, P.Miné and D.Vartsky, Nucl. Instr. and Meth. A327(1993)369.

[14] T.Francke and M.Suffert, Nucl. Instr. and Meth. A276(1989)263.

[15] I.M.Bronshtein and B.S.Fraiman, Secondary electron emission, (Nauka, Moscow, 1969), p.245, p.283-293 (in Russian).

[16] J.H.Conway and N.J.A.Sloane, Sphere Packing, Latticies and Groups, (Springer-Verlag, New York, 1988).

[17] P.M.Morse and H.Feshbach, Methodes of Theoretical Physics, part II, (McGraw-Hill Book, New York, 1953), p.1236.

[18] S.Majewski, G.Charpak, A.Breskin and G.Mikenberg, Nucl. Instr. and Meth. 217(1983)265.

[19] A.Breskin, A.Buzulutskov, R.Chechik, D.Vartsky, G.Malamud and P.Miné, Nucl. Instr. and Meth. A344(1994)537.

[20] R.Enomoto, T.Sumiyoshi and Y.Fujita, Nucl. Instr. and Meth. A343(1994)117.

[21] A.Borovick-Romanov and V.Peskov, Nucl. Instr. and Meth. A348(1994)269.

[22] L.Heroux, W.J.McMahon and H.E.Hinteregger, Appl. Opt. 5(1966)1338.

[23] B.Wassermann, S.Mirbt, J.Reif, J.C.Zink and E.Matthias, J. Chem. Phys. 98(1993)10049.

[24] A.Buzulutskov, A.Breskin and R.Chechik, The decrease of the LiF electron affinity by water adsorption, in preparation.

[25] G.Charpak, D.Lemenovski, V.Peskov and D.Scigocki, Nucl. Instr. and Meth. A310(1991)128.

[26] A.H.Sommer, Photoemissive Materials, (Robert E.Krager Publ., New York, 1980). 